\title{
Structural engineering in self-assembly of amphiphilic block copolymers with reactive additives: micelles, vesicles and beyond
}

\footnotetext{
Lei Jin, ${ }^{1}$ Chung-Hao Liu, ${ }^{3}$ Daniel Cintron, ${ }^{1}$ Qiang Luo, ${ }^{1}$ Mu-Ping Nieh, $, 2,3$ and Jie He ${ }^{* 1,3}$

${ }^{1}$ Department of Chemistry, ${ }^{2}$ Department of Chemical \& Biomolecular Engineering, and ${ }^{3}$ Polymer Program, Institute of Materials Science, University of Connecticut, Storrs, CT 06269, USA Email: jie.he@uconn.edu
}

(a)
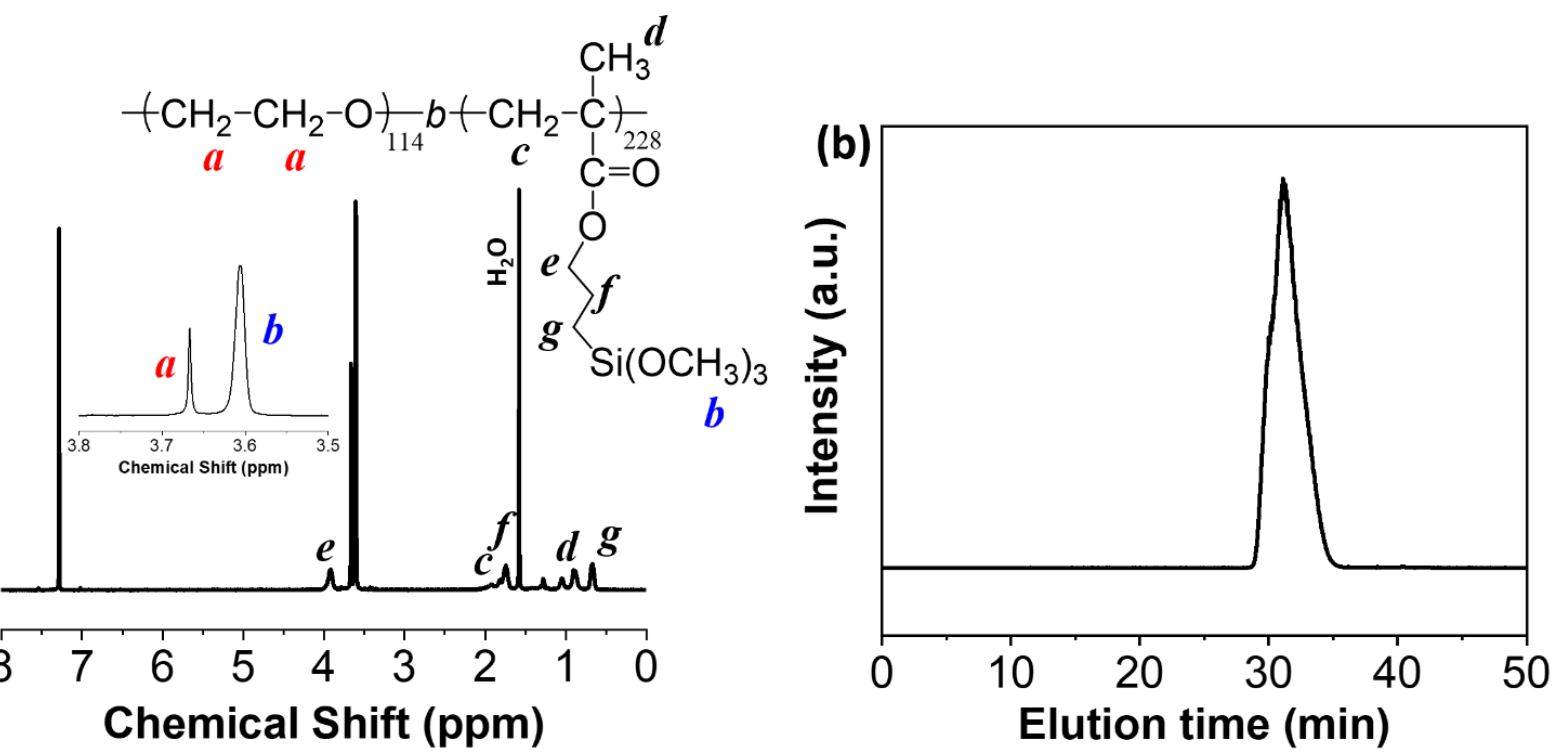

Figure S1. (a) NMR spectrum and (b) GPC elution curve of $\mathrm{PEO}_{114}-b-\mathrm{PTMSPMA}_{22}$. 

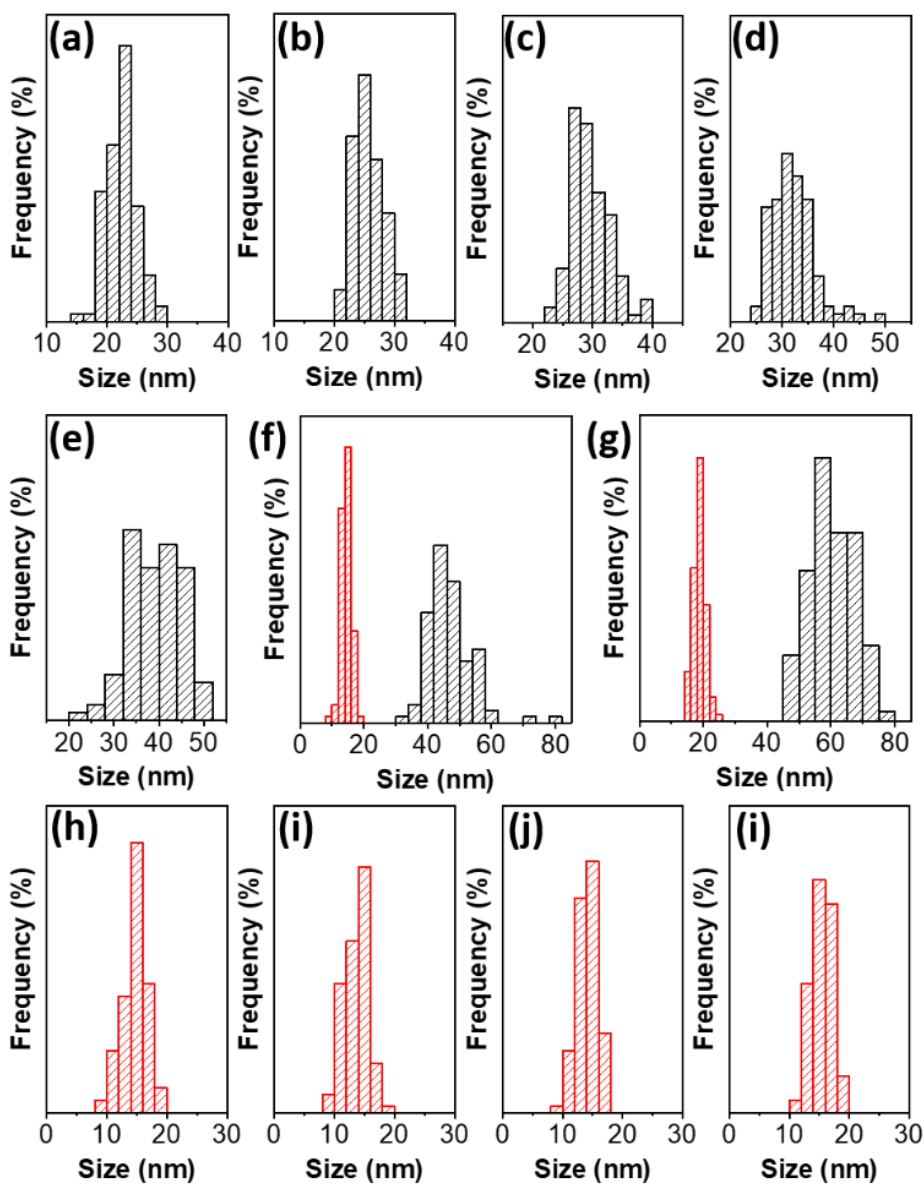

Figure S2. Size distribution of Si_1 to Si_11. Black: size of micelles or vesicles. Red: wall thickness of vesicles.
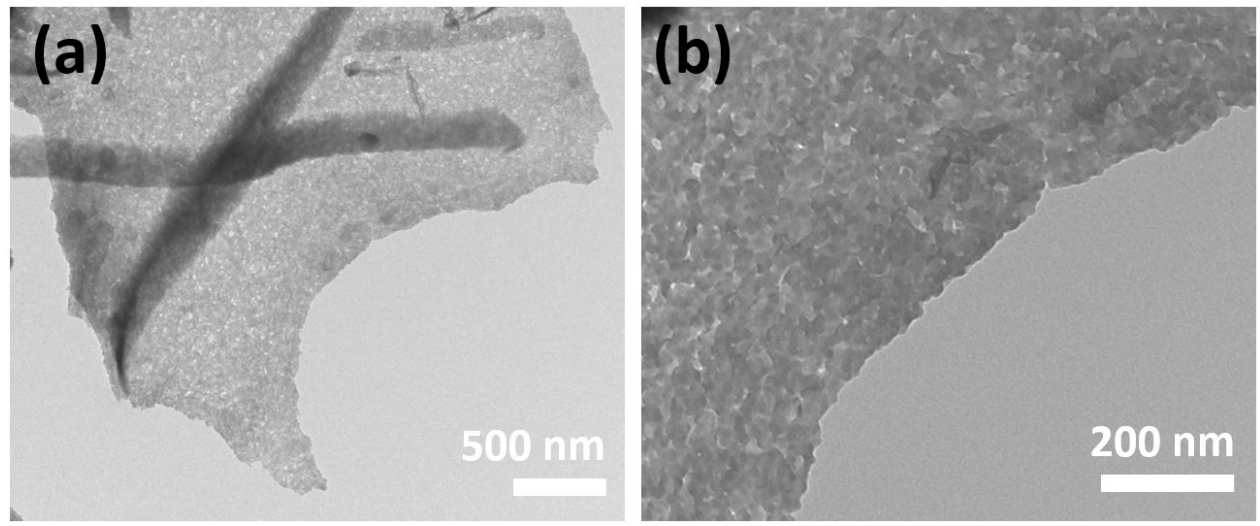

Figure S3. TEM images of Si-0 after calcination at $550{ }^{\circ} \mathrm{C}$. 
Table S1. Radius of gyration $\left(\mathrm{R}_{\mathrm{g}}\right)$ and hydrodynamic radius $\left(\mathrm{R}_{\mathrm{h}}\right)$ of different particles measured from

\begin{tabular}{lcccc}
\multicolumn{5}{c}{ SLS } \\
\hline No & Morphology & $\begin{array}{c}\mathbf{R}_{\mathbf{g}} \\
(\mathbf{n m})\end{array}$ & $\begin{array}{c}\mathbf{R}_{\mathbf{h}} \\
(\mathbf{n m})\end{array}$ & $\mathbf{R}_{\mathbf{g}} / \mathbf{R}_{\mathbf{h}}$ \\
\hline Si-0 & Micelles & 45.4 & 55.8 & 0.81 \\
Si-4 & Micelles and vesicles & 60.1 & 85.4 & 0.70 \\
Si-7 & Vesicles & 111.1 & 106.0 & 1.05 \\
Si-8 & Vesicles and complexed & 136.3 & 139.0 & 0.98 \\
Si-9 & vesicles & 138.1 & 140.0 & 0.99 \\
\hline
\end{tabular}

\section{Description of core-shell spherical model (form factor): ${ }^{1}$}

The intensity $I(q)$ for multi-shell vesicles is given by:

where

$$
I(q)=\frac{\text { scale }}{V} \times F(q)^{2}+\text { background }
$$

$$
F(q)=\frac{3}{V_{s}}\left[V_{c}\left(\rho_{c}-\rho_{s}\right) \frac{\sin \left(q r_{c}\right)-q r_{c} \cos \left(q r_{c}\right)}{\left(q r_{c}\right)^{3}}+V_{s}\left(\rho_{s}-\rho_{\text {solv }}\right) \frac{\sin \left(q r_{s}\right)-q r_{s} \cos \left(q r_{s}\right)}{\left(q r_{s}\right)^{3}}\right]
$$

where $V_{\mathrm{s}}$ and $V_{\mathrm{c}}$ are the volume of the whole particle and core, respectively. $r_{\mathrm{s}}$ and $r_{\mathrm{c}}$ are the radius of particles (radius plus thickness) and core radius, respectively. $\rho_{\mathrm{c}}, \rho_{\mathrm{s}}, \rho_{\text {solv }}$ are the scattering length density of the core, the shell and the solvent, respectively.

Here, we utilized the core-2-shell model to describe the $\mathrm{Si}-11$ and porous materials. The figures are shown in the Figure S4.

\section{(a) Si-11}

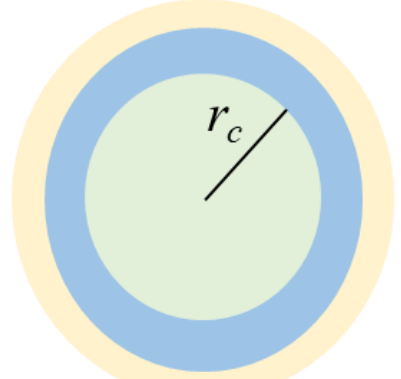

(b) Porous materials

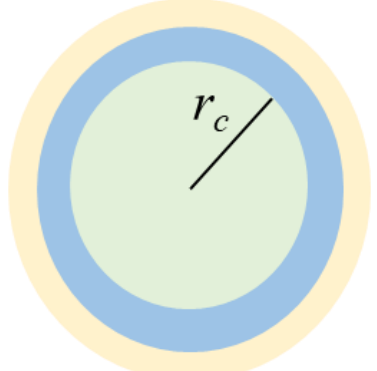

Figure S4. The core-2-shell model is applied to $\mathrm{Si}-11$ and porous materials.

Description of hard-sphere (HS) model (structure factor): ${ }^{2}$ This model is to describe the interparticle structure factor for spherical particles interacting through hard sphere interactions. The hard-sphere model uses the Percus-Yevick closure where the interparticle potential is

$$
\boldsymbol{U}(\boldsymbol{r})= \begin{cases}\infty & \boldsymbol{r}<2 \boldsymbol{R} \\ \mathbf{0} & \boldsymbol{r} \geq \mathbf{2} \boldsymbol{R}\end{cases}
$$


where $\mathrm{r}$ is the distance from the center of the sphere of a radius $R$.

The effective radius of the hard sphere, $R_{\text {eff }}$ in the HS model provides the insight to the possible closest

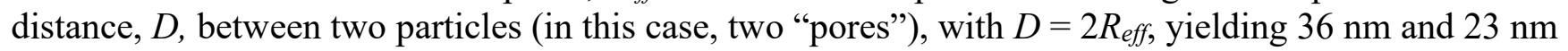
for $\mathrm{Si}-11$ and porous medium, respectively.

\section{Reference}

1. Guinier, A.; Fournet, G.; Yudowitch, K. L., Small-angle scattering of X-rays. John Wiley \& Sons, Inc.: 1955.

2. Percus, J. K.; Yevick, G. J., Analysis of Classical Statistical Mechanics by Means of Collective Coordinates. Phys. Rev. 1958, 110 (1), 1-13. 